\title{
Amniotic Band Syndrome with Cranial Anomalies; Review of Two Cases
}

\author{
Aydin Atis Alev*1, Acar Zuhat ${ }^{1}$, Topbas Selcuki Fitnat ${ }^{1}$, Gurbuz Sümeyye ${ }^{1}$, Ece Ermin, Acar Hicran ${ }^{2}$ \\ ${ }^{1}$ Obstetrics \& Gynecology, University of Health Sciences, Sisli Hamidiye Etfal Training and Research Hospital, Obstetrics \& Gynecology, Istanbul, \\ Turkey \\ ${ }^{2}$ Obstetrics \& Gynecology University of Health Sciences, Okmeydan Training and Research Hospital, Obstetrics \& Gynecology, Istanbul, Turkey
}

*Corresponding Author: Aydin Atis Alev, Obstetrics \& Gynecology, University of Health Sciences, Sisli Hamidiye Etfal Training and Research Hospital, Obstetrics \& Gynecology, Istanbul, Turkey.

\section{Received date: December 18, 2021; Accepted date: January 28, 2022; Published date: February 03, 2022}

Citation: Aydin A Alev, A Zuhat, Topbas S Fitnat, G Sümeyye, E Ermin, A Hicran. (2022) Barriers to Tracheostomy Weaning: A Case Report of Sialorrhea in a Patient with Persistent Seizure Activity and Head Injury. International Journal of Clinical Case Reports and Reviews. 10(4); DOI: $10.31579 / 2690-4861 / 198$

Copyright: (C) 2022 Aydin Atis Alev, This is an open access article distributed under the Creative Commons Attributiosn License, which permits unrestricted use, distribution, and reproduction in any medium, provided the original work is properly cited.

\begin{abstract}
Amniotic band sequence (ABS) results from in utero entrapment of fetal parts by fibrous bands, which leads to malformations that can affect multiple organ systems including the limbs, craniofacial regions and trunk, and central nervous system. These bands can adhere to anatomical structures of the fetus and cause vascular disruption which results in the amputation of the involved anatomic part. We present two cases of ABS both of which presented with craniofacial defects associated with ABS; one with apical encephalocel and extremity defects, the other one with schizencephaly with extremity defects and cleft lip \& palate. ABS may represent itself not only with extremity defects but atypical forms in cranial vault. ABS must be kept in mind and sonography must be detailed when rare deformities like schizencephaly and extremity defects identified.
\end{abstract}

Key words: amniotic band syndrome; amniotic band sequens; encephalocel; schizencephaly; limb defect

\section{Introduction}

Amniotic band sequence (ABS) is a congenital disorder presenting with fetal anomalies associated with fetal placental fibrous bands that may cause disruptions, deformations or malformations without a consistent anatomical pattern Amniotic band syndrome (ABS) is a prenatal complication with an incidence ranging from $1 / 1000$ to $1 / 15000$ in live births and 1/70 in stillbirths [1]. However, no known inheritance pattern has been reported. Although its etiology is not known, it is presumed that amniotic rupture during early pregnancy weeks lead to the development of multiple amniotic strands also called amniotic bands [2]. These bands can adhere to anatomical structures of the fetus and cause vascular disruption which results in the amputation of the involved anatomic part. Known Risk factors are Smoking (> 15 cigarettes/day) Exposure to cocaine and alcohol abuse, High glycemic index in the diet, Living in an attitudinal zone > 2000 meters above sea level, Obstetric factors, Attempted abortion, Uterine or abdominal trauma, Bicornuate uterus, Uterine fibroids, Ectopic pregnancy, hemorrhage during the first trimester of gestation, Intrauterine infection, Oligohydramnios, Intrauterine device removal, Chorionic villus sampling or amniocentesis. The cause of the amniotic rupture is mostly not clear. Constrictive rings, limb defects, neural or spine defects and craniofacial defects are the major classifications of ABS .Encephalocele, facial clefts and cleft lip and/or palate that are located non-midline atypical locations are among the craniofacial defects associated with ABS $[3,4]$.

Prenatal diagnosis of ABS can be challenging because of the variety of clinical presentations and the unclear etiology. Edema detected at the distal limbs with ultrasonography can indicate the presence of ABS. Prenatal detection can be as early as the first trimester if a major deformation or amputation is present [2,3]. However, the bands themselves can only be seen in few cases.

Here we present two cases of ABS detected at our tertiary maternal-fetal obstetrics clinic. Both cases presented with craniofacial defects associated with ABS and in both cases parents chose termination of pregnancy due to the severity of the anatomical defects. Informed consent were taken and approved in Local Ethics Committe accordingly.

\section{Case 1}

A 35 years old, g3 p2 pregnant patient at 22 weeks of gestation visited the perinatology outpatient clinic for a routine second trimester scan. Patient's medical anamnesis revealed no comorbidities. Her previous pregnancies were without any complications, where she delivered 
vaginally two healthy female newborns. During the current pregnancy she opted not to do the first and second trimester genetic screenings. Ultrasound scan was performed by an experienced perinatologist, who reported a single intrauterine fetus with biometric measurements in accordance with 22 weeks of gestation. An abnormal cranial anatomy with left ventriculomegaly was observed. Lateral ventricles were connected at subarachnoid space posteriorly, which was interpreted as schizencephaly. Anatomical scan of the fetal face revealed abnormalities with the absence of nasal bone and cleft lip and palate. Amniotic band syndrome with extremity defects were diagnosed. Fetal cranial magnetic resonance imaging (MRI) revealed cranial cleft in the left occipital region was reaching from the left ventricle to the subarachnoid space confirming the ultrasound diagnosis of schizencephaly. Cortex in this region was hypoplasic, bilateral occipital horns were dilated, cerebellum and vermis was hypoplasic related with dilated posterior fossa and partial corpus callosum agenesis was reported. Following the confirmation of diagnosis with MRI, parents opted for termination of the pregnancy. Medical fetocide was performed and labor was induced. A $555 \mathrm{gr}$ female fetus was delivered with limb defects (Figure 1,2,3).

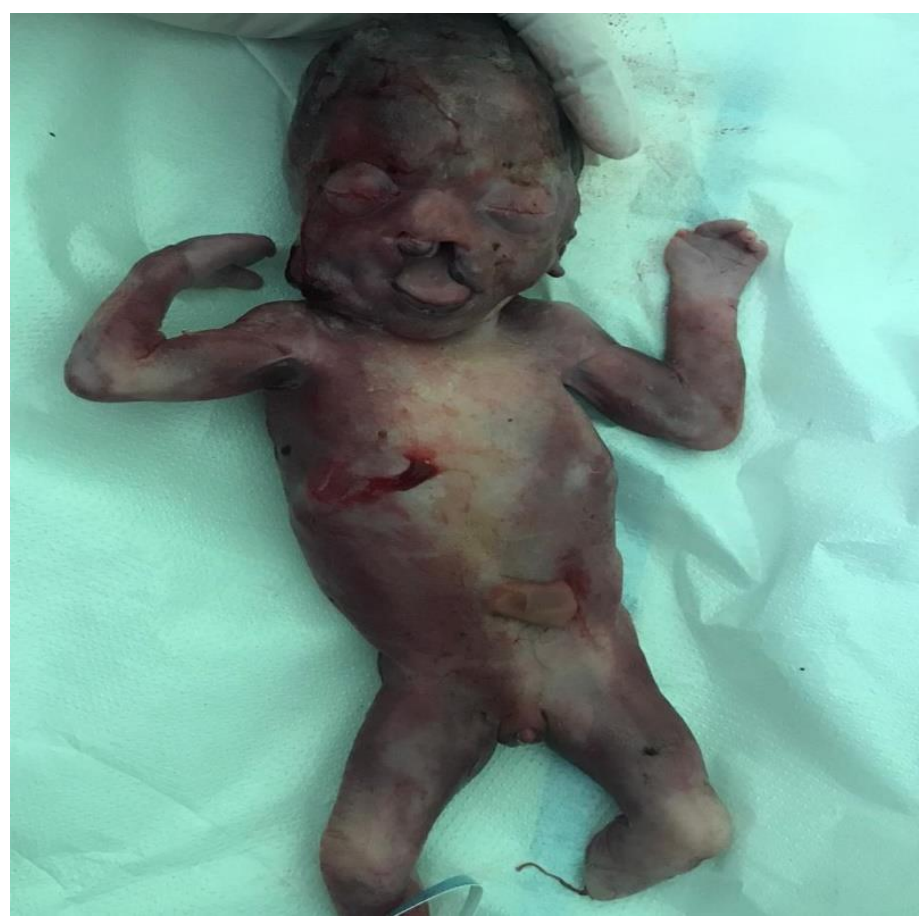

Figure1: Amniotic Band Sequence with Schizencephaly, postnatal abort

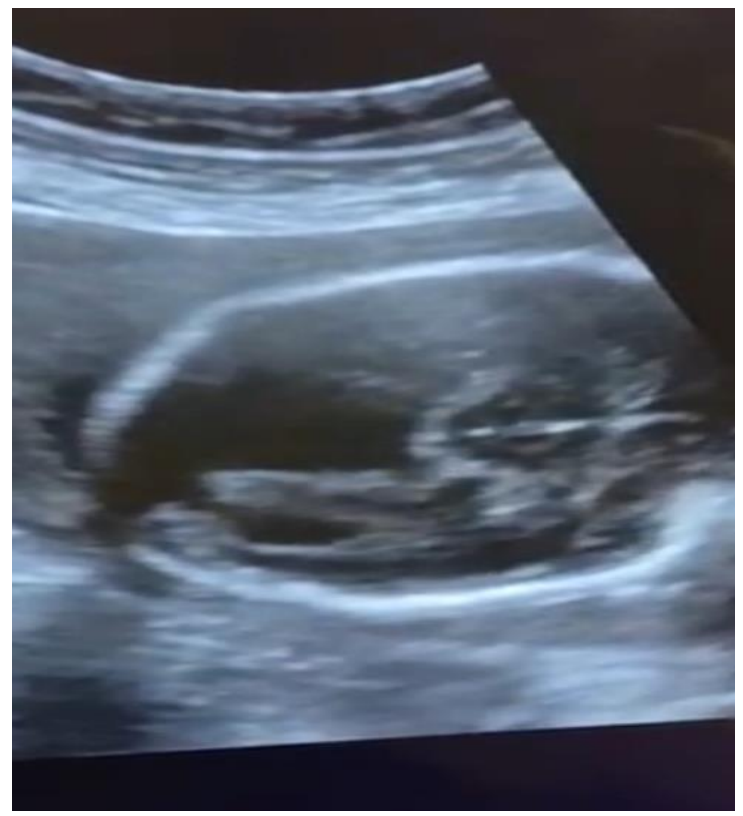

Figure 2: Fetal Sonography of Schizencephaly 


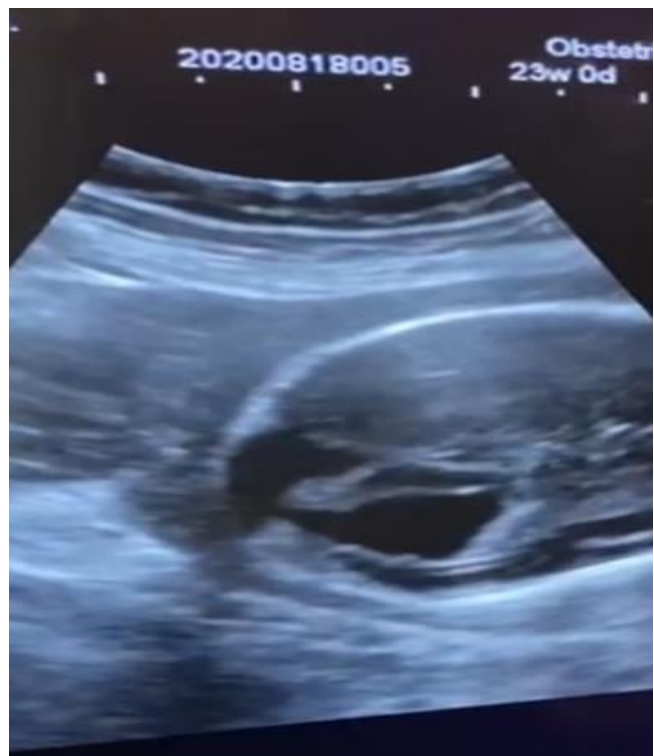

Figure 3: Fetal Sonography of Schizencephaly

\section{Case 2}

A 35 years old, gravidity 1 parity 0 pregnant patient at 14 weeks of gestation was referred with a suspected encephalocele. Patient had no history of any chronic disorders or operations and no known genetic disorders in her or her partner's family. First trimester ultrasound scan at $14^{\text {th }}$ weeks of gestation revealed encephalocele which is originating from $14 \mathrm{~mm}$ midline vertex defect deviated to left side $12 \mathrm{~mm}$ in length and left hand ulnar deviation and left foot pes equinavarus and left distal tubuler bone defect and accompanying left ulnar deviation were seen . There were lineer echogenic bands extending from cranium to extremities confirming amniotic band sequence was detected. Parents opted for termination of pregnancy. Labor was induced medically and patient aborted without any complications (Figure 4, 5).

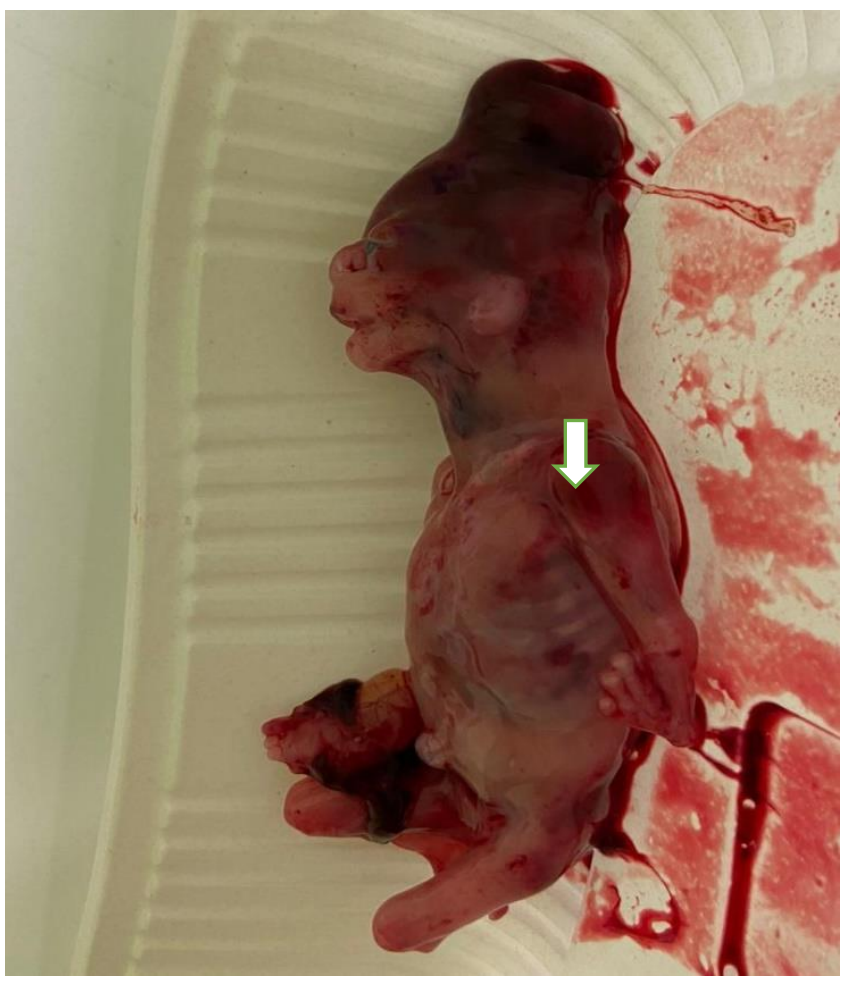

Figure 4: Amniotic Band Sequence with Encephalocel, postnatal abort 


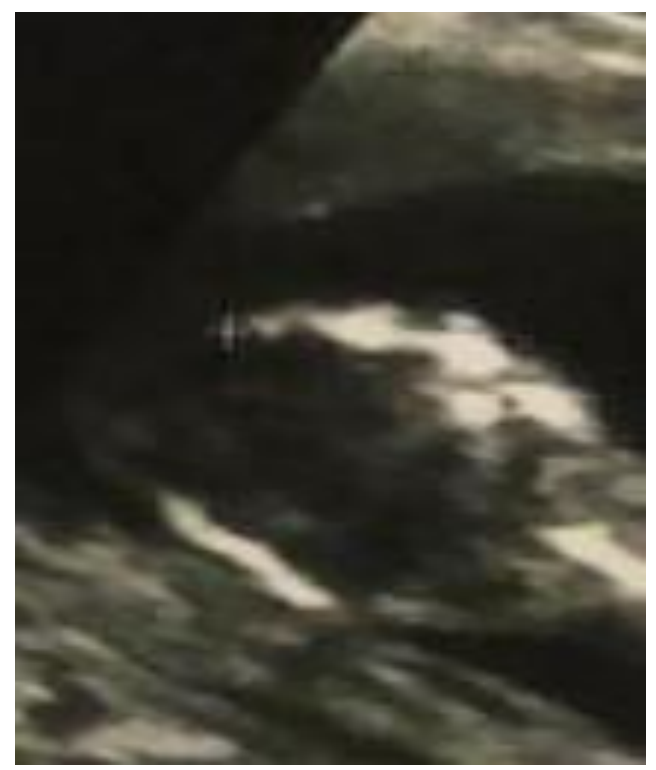

Figure 5: Encephalocel, usg view.

\section{Discussion}

Amniotic band sequence (ABS) results from in utero entrapment of fetal parts by fibrous bands, which leads to a collection of malformations that can affect multiple organ systems.

One of ABS etiologies defined by Romero et al. is early rupture of amniotic and chorionic membranes and the epithelial-mesenchymal transition was a factor that could contribute to ABS pathogenesis [6], second theory is by Van Allen et al. that internal or external events caused vascular accidents or had a negative impact on blood flow to the embryo because they interrupted the morphogenesis or destructed structures, whereas amniotic bands were a form of superficial necrosis [7] There is typically an asymmetric distribution of these defects; the most common manifestations involve limb deformities. Constriction ring defects of the extremities typically are present. These usually involve fingers or toes with or without edema of a distal extremity. These constriction rings can lead to amputation of limbs or digits and the appearance of syndactyly on ultrasound imaging (pseudosyndactyly) [4, 5]. Craniofacial deformities can include single orbital involvement, severe clefts that do not conform to a pattern of developmental clefts, severe nasal deformity, or asymmetric encephaloceles. This case report also reports amniotic band sequence associated with cranial anomalies one with an encephalocel and limb anomalies and the other with a schizencephaly with limb anomalies rather rare form.

All organ systems must be evaluated because there can be limb, craniofacial, or body wall abnormalities as ectopia cordis, gastroschisislike bowel herniation, omphalocele-like liver herniation, and bladder exstrophy [5].

Genetic evaluation in the setting of amniotic band sequence is not typically recommended. These cases are generally sporadic with no family history and no increased risk or incidence based on race or sex. Although a few familial cases have been reported, almost all cases are sporadic with no identified underlying genetic cause. If the diagnosis of amniotic band sequence is uncertain, chromosomal microarray analysis may be considered. A detailed ultrasound examination is recommended to identify the extent of the abnormalities.

There no guidelines for the management of ABS if detected during the prenatal period. Decision regarding the pregnancy and its management is taken based on the severity of the anatomical abnormality and the decision of the parents.Pregnancy termination is an option that should be discussed with all patients in whom a fetal anomaly is detected. Fetoscopic in utero lysis of bands is an investigational approach that has been reported $[4,5,8]$.

Diffrential diagnosis must be made with Body stalk anomaly that is characteristic with Fetal abdominal wall adherent to placenta, short umbilical cord, no cranial or limb defects, no evidence of fibrous bands or Specific craniofacial defects or abdominal wall defects: or with Amniotic sheets: Amnion wrapped around synechiae and a structurally normal, mobile fetus; importantly, amniotic band sequence should not be diagnosed in the absence of fetal abnormalities [3-5].

Craniofacial defects \& clefts are usually asymmetric and do not follow the anatomy of facial clefts; they extend from the lip and/or palate towards the skull and may or may not be connected to the brain malformation caused by the amniotic bands, although "normal" cleft lip and palate cases have also been reported like our first case having apical encephalocel and limb defects without cleft lip. Our case is characteristic with schizencephaly which is described a cleft extending from left ventricle in occipital region to subarachnoid space at the same side .There was also paranchimal loss and dysplasia nearby. Amniotic band sequens may present rarely with schizencephaly which must be kept in mind .Reported Head and neck lesions are encephalocele, exencefalia, acephaly, anencephaly/acrania, holoprosencephaly, microcephalus, ventriculomegaly and/or system hydrocephalus, septo-optic dysplasia, periventricular nodular heterotopia, polymicrogyria, cortical dysplasia with pachygyria, hypoplasia of the corpus callosum, craniosynostosis, myelomeningocele; in Face Asymmetry, agnathia, clefts Eyes, Orbital hypertelorism, eyelid coloboma, ectropion anophthalmia/microphthalmia ;in Nose Choanal atresia, Mouth Microstomia, high-arched palate, aglossia/microglossia, cleft lip and/or palate; in ears low ear implantation or rotation, poorly-differentiated pinna, melotia [8]

The antenatal diagnosis of amniotic adhesions has been associated with an adverse prognosis. Most craniofacial and body wall defects are incompatible with extrauterine life. In relation to amniotic bands, the most important fetal prognostic factor is perfusion of the distal portion of the affected limb. There have been reports of fetal death caused by umbilical cord strangulation by an amniotic band, although spontaneous resolution of constriction bands has also been reported and there is a chance of fetoscopic lysis by laser in mild forms [5,8]. 
Schizensefaly which is a rare congenital brain defect characterized by gray matter lined clefts of the cerebral mantle, has heterogeneous etiologies many of which are vascular disruptive in origin, they accompany non-CNS abnormality, over half of which could be classified as secondary to vascular disruption, including gastroschisis, bowel atresias, and amniotic band disruption sequence [5,9,10] schizencephaly in this case report also presented with amniotic band sequens as reported above. The causes of schizencephaly are heterogeneous but remain rather poorly understood. Established etiologies include the teratogens, warfarin, alcohol, and cocaine; the in-utero infections, cytomegalovirus, and herpes virus; maternal trauma of several types and monozygotic twin interactions All of these agents have as an underlying pathogenic mechanism, hypoxic ischemic vascular injury at critical times in neuronal development $[9,10]$

Amniotic band syndrome can vary in severity, depending on the organ systems that are affected; findings are typically asymmetric in distribution. The limbs are affected most commonly,. Patients can be reassured that recurrence is rare. Prognosis depends on the time of diagnosis (most cases are diagnosed post-natally), type and location of anomalies, and may vary from cosmetic to life-threatening consequences.

Declaration of interest: the authors have nothing to declare.

\section{Funding: none}

\section{References}

1. Kalousek DK, Bamforth S. (1988). Amnion rupture sequence in previable fetuses. Am J Med Genet. 31(1):63-73.
2. Gandhi M, Rac M. W. F \& McKinney J. (2019). Amniotic Band Sequence. American Journal of Obstetrics and Gynecology. 221(6):5-6.

3. Seeds JW, Cefalo RC, Herbert WN. (1982). Amniotic band syndrome. Am J Obstet Gynecol. 144(3):243-248.

4. Barzilay E, Harel Y, Haas J, et al. (2015). Prenatal diagnosis of amniotic band syndrome: risk factors and ultrasonic signs. J Matern Fetal Neonatal Med. 28:281-283.

5. Iqbal C. W, Derderian S. C, Cheng Y, Lee H \& Hirose S. (2014). Amniotic Band Syndrome: A Single-Institutional Experience. Fetal Diagnosis and Therapy. 37(1):1-5.

6. Romero-Valdovinos M, Galván-Montaño A, Olivo-Díaz A, et al. (2015). The amniotic band syndrome in the rat is associated with the activation of transforming growth factor-B. Am J Pathol. 185(8):2076-2082.

7. Van Allen MI, Curry C, Gallagher L. (1987). Limb body wall complex: I Pathogenesis. Am J Med Genet. 28(3):529- 548.

8. Eunice López-Muñoz, Luis E Becerra-Solano. (2018). An update on amniotic bands sequence. Arch Argent Pediatr. 116(3):409420.

9. Cynthia J Curry, Edward J Lammer, Verne Nelson, Gary M Shaw. (2005). Schizencephaly: heterogeneous etiologies in a population of 4 million California births. Am J Med Genet A. 137(2):181189.

10. Werler MM, Louik C, Mitchell AA. (2003). Epidemiologic analysis of maternal factors and amniotic band defects. Birth Defects Res Part A. Mol Teratol. 67:68-72.
This work is licensed under Creative Commons Attribution 4.0 License
To Submit Your Article Click Here: $\quad$ Submit Manuscript

DOI: $10.31579 / 2690-4861 / 198$
Ready to submit your research? Choose Auctores and benefit from:

$>$ fast, convenient online submission

$>$ rigorous peer review by experienced research in your field

$>$ rapid publication on acceptance

$>$ authors retain copyrights

$>$ unique DOI for all articles

$>$ immediate, unrestricted online access

At Auctores, research is always in progress.

Learn more https://auctoresonline.org/journals/international-journal-of-clinicalcase-reports-and-reviews 